\title{
Landscape and Flux Theory of Non-equilibrium Open Economy
}

\author{
Kun Zhang ${ }^{\mathrm{a}}$, Jin Wang,a,b,d,* \\ ${ }^{a}$ State Key Laboratory of Electroanalytical Chemistry, Changchun Institute of Applied \\ Chemistry, Chinese Academy of Sciences, Changchun, Jilin,130022, P.R.China \\ ${ }^{b}$ Department of Chemistry and Physics, Stony Brook University, Stony Brook, New York, \\ 11794. \\ ${ }^{c}$ Department of Applied Mathematics and Statistics, Stony Brook University, Stony \\ Brook, New York, 11794. \\ ${ }^{d}$ Harriman School of Business Management,Stony Brook University, Stony Brook, New \\ York, 11794.
}

\begin{abstract}
The economy is open and never in true equilibrium due to the exchanges with outside. However, most of the quantitative studies have been focused on the equilibrium economy. Despite of the recent efforts, it is still challenging to formulate a quantitative theory for uncovering the principles of non-equilibrium open economy. In this study, we developed a landscape and flux theory for non-equilibrium economy. We quantify the states of economy and identify the multi-stable states as the basins of attractions on the underlying landscape. We found the global driving force of the non-equilibrium economy is determined by both the underlying landscape gradient and the curl probability flux measuring the degree of non-equilibriumness through the detailed balance breaking. The non-equilibrium thermodynamics, the global stability, the optimal path and speed of the non-equilibrium economy can be formulated and quantified. In the conventional economy, the supply and demand usually has only one equilibrium. By considering nonlinear supply-demand dynamics, we found that both bi-stable states and limit cycle oscillations can emerge. By shifting the slope of demand curve, we can see how the bi-stability transforms to the limit cycle dynamics and vice versa. By parallel shifting the demand curve, we can also see how the monopoly, the competition, and the bistable monopoly and competition states emerge
\end{abstract}

*To whom correspondence should be addressed. E-mail: jin.wang.1@stonybrook.edu 
and transform to one other. We can also see how the mono-stable monopoly, the limit cycle and the mono-stable competition states emerge and transform to one another.

Keywords: supply-demand, non-equilibrium economy, landscape.

\section{Introduction}

In equilibrium economy, the interplay and balance between supply and demand becomes the key. The corresponding models have been widely used to illustrate how the balance of the price from the demand and production supply is determined. Demand is determined by many factors such as price, expected price, government policies, income level, advertisement, numbers of consumers etc. Supply is determined by also many factors such as price, price of other related products, expected price, government policies, cost and technology etc. A static single equilibrium can often be achieved for price when the supply and demand reach the balance. When the supply and demand change, the effects on the dynamics of the price and production quantity are usually described by the dynamical analysis based on the shift of the single equilibrium.

The "invisible hand" suggested by Adam Smith [1] can efficiently allocate resources in many free markets. The concept inspired Leon Walras to establish the existence of competitive general equilibrium for economy with linear supply and demand function [2]. Then Pareto defined optimality and established the coexistence of optimality and Walrasian general economic equilibrium. The theory was carried forward and extended.[3, 4]

Some economists have recognized the possibility of multiple equilibria for the partial equilibrium case in early times $[2,5]$. Such cases can give stable and unstable equilibria. In particular, Marshall considered that there can be an alternate tendency between stable and unstable equilibria. A small shift in a parameter can lead to a very significant change or transformation. As a result, the system can jump from mono-stable equilibrium to another one quite far away through an unstable equilibria. Furthermore, the idea of multiple oligopoly equilibria was also emerged. Joan Robinson first considered the pure monopoly case due to the possibility of upward slope of marginal revenue curves in a world of segmented markets [6]. Wald also exhibited this possibility for the Cournot case with marginal revenue of upward slope. Then Bonanno explored the Robinson type case with the smoothly shifted 
demand curves and fixed cost curves in a catastrophe theory [7].

In classical economics, economists mainly focused on the theory of static or equilibrium economy rather than non-equilibrium economy or dynamics. The equilibrium study is in general simpler and may serve as a useful preparation for the more difficult non-equilibrium and dynamics. In late nineteenth century, the neoclassical economists provided fruitful mathematical analysis of the equilibrium in an idealized market economy. With the progress of economy, some prominant economists started to realize the importance of nonequilibrium economics and the truly dynamic economy. Marx argued that there would be cycles in employment and income shares in a highly simplified economy composed of capitalists and workers. The famous Goodwin's model of cyclical growth was inspired from Marx and provided a cyclical vision of economy $[8,9]$. The defect of the equilibrium Say's Law/Walras's Law is that the role of credit is ignored [2]. The depiction of the equilibrium economic theory is the "general gluts" (as an excess of supply in relation to demand) and depressions are impossible because of the spontaneous equilibrium. Schumpeter, Marx and Minsky pointed out that the depressions and "general gluts" are possible once the fallacy is removed. Schumpeter focused upon the role of entrepreneurs in capitalism and presented the vision of cycles driven by entrepreneurs search for profit $[8,10,11]$. Fisher's debt-deflation theory claimed the viewpoint of cycles and potential collapse caused by private debt[12]. Keynes also suggested that the economy cannot spontaneously attain equilibrium due to uncertainty and fragility of expectations [13]. In summary, those kinds of the economic cycle and crisis phenomena cannot be perfectly comprehended in a statics and equilibrium system. More recently, economists such as Steve Keen also suggested the idea and concept of nonequilibrium economics[14, 15].

As discussed, in the conventional economy, the supply and demand have often been described as having a monotonic trend. Higher price is accompanied with higher production quantity for supply. On the other hand, lower price is accompanied with higher demand in quantity for demand. This is often under the assumption of the complete competition market and close equilibrium economy. However, in reality, incomplete market competition, open non-equilibrium economy rather than closed economy, inflation and under-employment, and overproduction etc are present and can alter the simple monotonic trend to be nonlinear and non-monotonic. As a result, the multiple stable states can emerge. In some cases, the limit cycle oscillation can appear. The underlying trend can no longer be determined by the simple 
shift of the single equilibrium as in the conventional supply-demand dynamical analysis in economy. The challenges are then to formulate a quantitative theory for non-equilibrium economy to address the issues of how to globally and quantitatively describe the multiple stable states and limit cycle oscillations in a non-equilibrium economy, how to identify the driving forces for non-equilibrium economy, and how to describe the dynamical process (characterized by paths and speed) from one state to another for the economy in the more general and realistic non-equilibrium cases other than the idealized simplified equilibrium cases.

In this work, we developed a landscape and flux theory to meet the above challenges for describing the general dynamics of non-equilibrium economy. We first define the economic state as determined by the collection of the specific values of the underlying state variables such as price, production quantity and many others. Then the state can be described as one point in this multidimensional space of variables. The dynamics of economy can be seen as the evolution from one state to another in this multidimensional space. Since there are in principle many such states in the multi-dimensional state space, not every state is equally probable. We quantify the states of economy by identifying the multi-stable states as the basins of attractions on the underlying probabilistic landscape with higher probabilities. The market in a specific economic state can fluctuate around specific price and production. Once the fluctuations of price and production are large enough to overcome the barrier of underlying landscape, the market can enter into another state of economy from the current state and achieve a new steady state. It is worthwhile emphasizing that our approach of quantifying nonequilibrium economic dynamics with states and state transitions in between is in contrast to the conventional quantification of economic dynamics as equilibrium shift.

We uncovered the driving forces of economy, one is from the underlying landscape gradient derived from the steady state probability distributions and the other is from the curl probability flux which measures the degree of detailed balance breaking or the exchange with outside for open nonequilibrium economy. The dynamics of economy thus resembles an electron moving in an electric (landscape gradient) and magnetic field (curl flux). Notice the conventional equilibrium economic dynamics does not have this landscape perspective. Furthermore, the conventional equilibrium dynamics in complex systems only has the landscape gradient as the sole driving force. It does not have the curl flux component. However, the economy is an open 
and non-equilibrium system. That is why both landscape gradient and curl flux are playing the key roles in determining the general non-equilibrium economic dynamics.

In the conventional equilibrium economy, the supply and demand often have only one equilibrium point and the dynamics is described as the shift of the equilibrium. By considering nonlinear supply-demand dynamics in open non-equilibrium economy, we found that both bi-stable states and limit cycle oscillations can emerge. In this model, the bi-stability corresponds to the coexistence of the competition and monopoly/oligopoly markets while the limit cycle corresponds to stable periodic oscillation through the competition and monopoly/oligopoly states. Bi-stable states can be quantified in the landscape picture as two basins of attractions, the depths of which represent probabilities of the states. We quantify the kinetic transition time between the bi-stable basins of attractions on the landscape. This gives a measure for the global stability of the bi-stable supply-demand economy. We also correlate the kinetic time with the landscape topography through the barrier height between the basins of attraction. Both the landscape topography and kinetic transition time can be used to measure how difficult it is for the state to change from one to the other (competition and monopoly/oligopoly), or in other words the global communication capability. We quantify the dominant paths between the basins of attractions of competition and monopoly/oligopoly to uncover the underlying kinetic processes from one stable state to anther. The paths are irreversible and do not always go through the saddle point between the landscape basins. This is due to the presence of the other driving force of the dynamics, the curl flux in addition to the landscape gradient.

We found the underlying landscape of limit cycle dynamics through the competition and monopoly/oligopoly states of the supply-demand economy has a irregular Mexican hat shape. Outside the oscillation cycle ring, the dynamics are attracted to the ring mainly by the landscape gradient. Once on the oscillation ring, the driving force for the limit cycle is then determined by the curl flux along the cycle and the dragging force is determined by the local basins and barriers on the cycle. Kinetic rates escaping from the cycle to the center island can be used to measure the global stability of the limit cycle oscillation for the supply-demand economy. This correlates with the barrier height between oscillation ring and the center island. We also estimated the energy consumption or dissipation required in the processes within the states and state transformations, such as bi-stability and limit cycle. We 
found energy consumption is correlated with the flux. Therefore, the flux is the manifestation of the energy consumption to maintain the stability of the open non-equilibrium economy. We further found the period and coherence of the limit cycle is determined by both the curl flux and the local barrier heights along the cycle.

By shifting the slope of the demand curve, we can see how the underlying economic dynamics in terms of the state changes: that is how the bi-stability of competition and monopoly transforms to the limit cycle dynamics and vice versa. By parallel shifting of the demand curve, we can see how the monopoly state is emerged and transformed to the coexistence of monopoly and competition bistable states, and furthermore, how the bistable states are transformed to the competition state, and vice versa. We can also see how the mono-stable monopoly state changes to the limit cycle oscillation state and how the oscillation is further changes to the mono-stable competition state, vice versa.

\section{Landscape and Flux Theory of Non-equilibrium Open Economy}

The dynamics of the economic model have often been studied by a set of ordinary differential equations of various economic variables, i.e. supply and demand etc. The equations always can be formulated in the vector form $\mathrm{d} \mathbf{x} / \mathrm{d} \mathbf{t}=\mathbf{F}(\mathbf{x})$, where the $\mathrm{N}$ dimensional vector $\mathbf{x}$ represents the $\mathrm{N}$ different economic variables and the vector $\mathbf{F}(\mathbf{x})$ is the driving force of the economic system. In the conventional economical model of supply and demand, only one equilibrium emerges. For the general nonlinear non-equilibrium dynamical economic systems, the dynamical behavior in principle can give distinct different global features such as multi-stability and limit cycle, chaos, and so on in addition to the mono-stability.

While following trajectory evolutions can give local details, global feature are often hard to see from individual trajectories. There is a simple global description of the economical system dynamics. When the driving force $\mathbf{F}(\mathbf{x})$ can be represented as the negative gradient of a potential function. This corresponds to the equilibrium dynamics often encountered in physics and chemistry. However, the force vector $\mathbf{F}(\mathbf{x})$ in economy does not in general satisfy the gradient condition for systems of more than one dimension. Therefore, the equilibrium landscape ideas and its corresponding dynamical description can not be applied to the economic systems. Then the challenge 
becomes how to quantitatively describe the global non-equilibrium economic dynamics?

In the past, many efforts have been focused on local stability and dynamical analysis for the corresponding non-linear models of economy [4, 16, 17]. But these approaches do not give a global description of the stability and associated dynamics.

Furthermore, the fluctuations of the economic environment and economic variables are present and can be significant for the relevant activity in economy. Therefore more appropriate model to describe the economic dynamics is the stochastic dynamics instead of the deterministic dynamics. The economic systems that are modelled by the ODEs for deterministic dynamics can then be replaced by a set of stochastic dynamics equations

$$
\frac{\mathrm{d} \mathbf{x}}{\mathrm{d} t}=\mathbf{F}(\mathbf{x})+\eta
$$

where $\eta$ is Gaussian white noise term with zero mean and its autocorrelation function is $\langle\eta(\mathbf{x}, t) \eta(\mathbf{x}, 0)\rangle=2 D \delta(t)$. D is the diffusion coefficient. It characterizes the intensity of the economic environmental and variable fluctuations.

\section{Landscape and Flux of Non-equilibrium Open Economy}

The stochastic systems are not globally described by the individual stochastic change of state but by the state probabilities. The dynamics is not globally described by a single trajectory but by the probability distribution evolution. The probability evolution of the stochastic system satisfies the Fokker-Planck equation in the continuous representation [18].

$$
\partial P / \partial t=-\nabla \cdot \mathbf{J}=-\nabla \cdot[\mathbf{F} P-\nabla \cdot(\mathbf{D} P)] .
$$

Here $\mathbf{J}$ is the probability flux $\mathbf{J}=\mathbf{F P}+\mathbf{D} \nabla \mathbf{P}$. The physical meaning of the above equation is the conservation law of probability. It shows that the change rate of of the local probability is equal to the flux entering or exiting the system.

If the underlying economic system reaches a steady state, the above equation can be written as $\partial P_{s s} / \partial t=-\nabla \cdot \mathbf{J}_{\mathbf{s s}}=\mathbf{0}$. There are two possibilities. The first one is $\mathbf{J}_{\mathbf{s s}}=\mathbf{0}$. The zero flux leads to the detailed balance that the economic system is in equilibrium without net in or out flux from the exchange 
from outside. According to the definition of flux, we find $\mathbf{F}(\mathbf{x})=-\mathbf{D} \cdot \partial \mathbf{U} / \partial \mathbf{x}$ where $U=-\ln P_{s s}$ is the equilibrium potential and $P_{s s}$ here represents the equilibrium probability distribution. In fact, this is the equilibrium Boltzmann law. In other words, the driving force for the equilibrium economic dynamics can be expressed as a gradient of a potential U. The equilibrium economic system follows gradient dynamics which is controlled by only the gradient of the potential or energy U. The global feature of the system and dynamical stability are determined by the underlying potential. The equilibrium dynamics of the economic system is in analogy with the case of an electron moving in electric field. This economic system is in equilibrium and therefore a closed one with no exchange of energy or information with external environments.

There exists another possibility for the steady state. The flux of the steady state is not equal to zero. Since there is a non-zero net flux, the detailed balance is broken and the system is in non-equilibrium state. We can see the flux measures the degree of non-equilibriumness of how far the system is from the equilibrium state. We can then decompose the driving force into three terms $\mathbf{F}=-\mathbf{D} \cdot \partial \mathbf{U} / \partial \mathbf{x}+\mathbf{J}_{\mathbf{s s}} / \mathbf{P}_{\mathbf{s s}}+\nabla \cdot \mathbf{D}$ where $\mathbf{U}$ is non-equilibrium potential related to the steady state probability by $U=-\ln P_{s s}[19,20]$. The flux has a curl nature due to the divergence-free steady state probability flux $J_{s s}$. The global nature and stability can then be described by the potential landscape $U$ since it is directly related to the steady state probability. The divergence of the diffusion tensor characterizes the inhomogeneity of the fluctuations. The driving force for the general non-equilibrium dynamics are determined by both the landscape gradient and curl flux force[21, 22, 23]. The general non-equilibrium dynamics is analogous to an electrons moving in both the electric and magnetic fields. For the general non-equilibrium economic systems, the landscape and flux can quantify the invisible hands of the market globally, as the driving force of the dynamics for controlling the production quantity and price. Therefore, we transform the local description of economic dynamics from each individual trajectories to global description of state transitions from one basin to another under the underlying landscape and flux.

\subsection{Optimal Kinetic Paths of Non-equilibrium Open Economy}

To quantify the dynamical processes in economy, we can also represent the dynamics with the condition probability from initial state $\mathbf{x}_{\mathbf{i}}$ at $\mathrm{t}=0$ to 
the final state of $\mathbf{x}_{\mathbf{f}}$ at time $\mathbf{t}$, by the Onsager-Machlup functional [21] as

$$
\begin{aligned}
P\left(\mathbf{x}_{\mathbf{f}}, t, \mathbf{x}_{\mathbf{i}}, 0\right) & =\int D \mathbf{x} \operatorname{Exp}\left[-\int d t\left(\frac{1}{2} \nabla \cdot \mathbf{F}(\mathbf{x})+\frac{1}{4}(d \mathbf{x} / d t-\mathbf{F}(\mathbf{x})) \cdot \frac{1}{\mathbf{D}(\mathbf{x})} \cdot(d \mathbf{x} / d t-\mathbf{F}(\mathbf{x}))\right)\right] \\
& =\int D \mathbf{x} \operatorname{Exp}[-S(\mathbf{x})]=\int D \mathbf{x} \operatorname{Exp}\left[-\int L(\mathbf{x}(t)) d t\right]
\end{aligned}
$$

where Dx denotes the sum over all possible paths from $\mathbf{x}_{\mathbf{i}}$ at $t=0$ to $\mathbf{x}_{\mathbf{f}}$ at time $\mathrm{t}$ and $\mathrm{D}(\mathrm{x})$ is the diffusion coefficient. The exponential factor contributes to the weight of each path. The total probability from the initial state to the final state is equal to the sum of different weights from every possible path. The $L(\mathbf{x}(\mathbf{t}))$ is the lagrangian and $S(\mathbf{x})$ is the associated action function or the weights of each path. Notice each path gives different contribution. Except for dominant path, the other sub-leading path contributions are often smaller and can be ignored because they are exponentially weighted. Further, we can identify the dominant path as the optimal economic path in our case. When the driving force $\mathbf{F}$ for economy is a gradient, the term $\int \mathbf{F} \cdot \mathbf{d x}$ in the weight functional above is a constant depending only on endpoint. If the driving force $\mathbf{F}$ is not purely a gradient, then the $\int \mathbf{F} \cdot \mathbf{d x}$ is no longer a constant and it influences the dynamics. As a consequence, the forward path and backward path will not be identical because of the curl flux component of the force. In other words, the corresponding economic pathways are irreversible. The curl flux breaking the detailed balance is the origin of the optimal kinetic paths deviating from the steepest descent one in nonequilibrium system. From the optimal path, we can also quantify the kinetic rate or speed from one state to another directly [21].

\subsection{Non-equilibrium Thermodynamics and Dissipation in Non-equilibrium Open Economy}

The non-equilibrium system is an open one with constant exchanges in energy and information to the environments. The system will have energy consumption and generate dissipation. The dissipation is a global physical characteristic of the nonequilibrium system. It is a distinctive nature of the nonequilibrium system. In fact, it can be used also as a measure of the non-equilibriumness of how far the system is from the equilibrium state. In the steady state of non-equilibrium system, the energy dissipation is closely related to the entropy production rate[20, 24]. The system entropy is known as $S=-\int P(\mathbf{x}, \mathbf{t}) \ln \mathbf{P}(\mathbf{x}, \mathbf{t}) \mathbf{d x}$. By differentiating the above function, the change rate of the system entropy can be 
given as, $\dot{S}=\int\left(\mathbf{J} \cdot \mathbf{D}^{-\mathbf{1}} \cdot \mathbf{J}\right) / \mathbf{P d x}-\int\left(\mathbf{J} \cdot \mathbf{D}^{-\mathbf{1}} \cdot(\mathbf{F}-\nabla \cdot \mathbf{D})\right) \mathbf{d} \mathbf{x}$, where $\int\left(\mathbf{J} \cdot \mathbf{D}^{-\mathbf{1}} \cdot \mathbf{J}\right) / \mathbf{P d x}=\mathbf{e}_{\mathbf{p}}=\dot{\mathbf{S}}_{\text {tot }}$ denotes the entropy production rate, it represents the total entropy change rate (including both system and environment) and $\int\left(\mathbf{J} \cdot \mathbf{D}^{-\mathbf{1}} \cdot(\mathbf{F}-\nabla \cdot \mathbf{D})\right) \mathbf{d} \mathbf{x}=\mathbf{h}_{\mathbf{d}}=\dot{\mathbf{S}}_{\text {env }}$ represents the mean rate of the heat dissipation, the entropy change rate from the environment. When the system reaches a steady state, the change rate of the system entropy is zero. Therefore, entropy production rate of the total entropy change rate from the system and the environment is equal to the heat dissipation from the environment. As we can see, the energy dissipation quantified by entropy production $e_{p}$ and $h_{d}$ is correlated directly to the curl flux $\mathbf{J}$. The equations can also be written as $\dot{S}_{t o t}=\dot{S}+\dot{S}_{e n v}$. This gives the first law of non-equilibrium thermodynamics. The entropy production is always larger or equal to zero. This gives the second law of non-equilibrium thermodynamics. [20] The first law of the non-equilibrium thermodynamics for non-equilibrium economy can also be formulated by the following equation: epr $=-\dot{F}+Q_{h k}$ where entropy production (epr) measuring the energy cost or assumption of the whole system and the environments is equal to the free energy $-\dot{F}$ relaxation to the steady state and the house keeping heat $Q_{h k}$ for maintaining the steady state. Here the free energy relaxation is directly related to the relative probability landscape gradient $\dot{F}=-\partial_{i} \ln \left[P(x, t) / P_{s s}(x)\right]$ where $P(x, t)$ and $P_{s s}(x)$ are the time dependent probability and the steady state probability respectively. The house keep heat is directly related to the curl flux by: $Q_{h k}=T<J_{i}^{s s} / P_{s s}(x) D_{i j}^{-1} J_{j}^{s s} / P_{s s}(x)>$ where $J_{i}^{s s}$ and $J_{j}^{s s}$ are the curl probability fluxes of $\mathrm{i}$ and $\mathrm{j}$ components, $P_{s s}(x)$ is the steady state probability and $D_{i j}^{-1}$ is the inverse diffusion matrix. [20]. This form of the first law of the non-equilibrium thermodynamics for non-equilibrium economy for the total entropy production as the sum of the free energy relaxation originated from the relative landscape gradient and the house keeping heat originated from the curl flux at the thermodynamic level is a direct reflection or manifestation of the total driving force decomposition as the sum of the landscape gradient and the curl flux for determining the dynamics at the dynamics level.[20]

\subsection{Global Stability and Lyapunov function for Non-equilibrium Open Econ- omy}

In order to study the global stability of economy model, we need to search for the Lyapunov function of the system. First of all, we consider the small noise limit $D \rightarrow 0$. In this case, we can expand the potential $U(\mathbf{x})$ with respect to the diffusion coefficient $D$ as following: $U(\mathbf{x})=$ 
$\left[\sum_{k=0}^{\infty} D^{k} \phi_{k}(\mathbf{x})\right] / D=\phi_{0}(\mathbf{x}) / D+\phi_{1}(\mathbf{x})+D \phi_{2}(\mathbf{x})+\cdots$. We can substitute the equation into the Fokker-Planck equation and compare the coefficients of every term on two sides of the equation in the order of diffusion $D^{-1}, 1, D, D^{2} \ldots$ Consequently, we can find a set of differential equations of $\phi_{i}(\mathbf{x})$ in the order of $D^{-1}, 1, D, D^{2} \ldots$ Through solving the equations, we can obtain $\phi_{i}(\mathbf{x})$. Particularly, $\phi_{0}(\mathbf{x})$ at the lowest order of $D^{-1}$ satisfies the following equation,

$$
\mathbf{F} \cdot \nabla \phi_{0}+\nabla \phi_{0} \cdot \mathbf{D} \cdot \nabla \phi_{0}=0
$$

The equation is called the Hamiltonian-Jacobian equation. On the basis of the following evolution equation for $\phi_{0}$,

$$
d \phi_{0}(\mathbf{x}) / d t=\nabla \phi_{0} \cdot \mathbf{F}=-\nabla \phi_{0} \cdot \mathbf{D} \cdot \nabla \phi_{0} \leq 0 .
$$

We can further see that $\phi_{0}$ monotonously decreases with a deterministic trajectory for semi-positively defined diffusion matrix. With the evolution of the system, it finally reaches the attractor states. Because of the monotonic decline property, $\phi_{0}$ is a Lyapunov function of system. It is important to find the Lyapunov function. Through the Lyapunov function, we can quantify and understand the global stability for the dynamical non-equilibrium economic systems.[23]

\subsection{Coherence}

In the limit cycle oscillation economic model, the robustness of the oscillation can be quantified by the phase coherence $\xi$, which denotes the degree or the persistence of periodicity of the time evolution of a given variable[25]. The phase coherence is defined with the following method. $N(t)=n_{1}(t) e_{1}+n_{2}(t) e_{2}$ is the vector at time t. $n_{1}(t)$ and $n_{2}(t)$ are the price and quantity of goods. $e_{1}=(1,0)$ and $e_{2}=(0,1)$ are the unit vectors. Then $\phi(t)$ is the phase angle between $N(t)$ and $N(t+\tau)$, where $\tau$ is smaller than the deterministic period and larger than the fast fluctuations. The coherence $\xi$ can be obtain, $\xi=\frac{2 \sum_{i} \theta(\phi(t)) \phi(t)}{\sum_{i}|\phi(t)|}-1$, where $\theta(\phi)=0$ when $\phi(t) \leq 0$, and $\theta(\phi)=1$ when $\phi(t)>0$, and sums contain every time steps of the trajectory. $\xi \approx 0$ represents the system moves stochastically. If $\xi$ is close to 1 , the oscillation is mostly coherent. The more periodic the evolution is, the larger the coherence $\xi$ is. 


\section{Supply and Demand Model}

In order to apply our landscape and flux theory of non-equilibrium economy, we investigate the supply and demand model in economy. Supply and demand are the very cores of economic concepts. The studies of the supplydemand model play remarkable role in the economy. We consider a simple model for the dynamics of price and quantity in a single market as follows $[5,16,26]$ :

$$
\begin{aligned}
\frac{\mathrm{d} P}{\mathrm{~d} t} & =\alpha(F(P)-Q) \\
\frac{\mathrm{d} Q}{\mathrm{~d} t} & =\beta(P-C(Q))
\end{aligned}
$$

where $\mathrm{P}$ denotes the price and $\mathrm{Q}$ denotes the quantity. $\alpha$ and $\beta$ denote speed of adjustment. The first equation represents that the change rate of price is proportional to the excess demand $F(P)$ over supply quantity. The second equation represents the change rate of quantity is related to the excess of price over cost $C(Q)$. In conventional studies, the excess demand and cost function often follow the linear dependence on the underlying variables. As a result, there is only one stable fixed point which represents the equilibrium of demand quantity and supply price in the goods market.

Obviously this is an oversimplified ideal situation. In order to further study the more general and realistic cases, we consider a nonlinear cost function $C(Q)$ in the differential equations $[16,17]$. The specific equations are written as:

$$
\begin{aligned}
& \frac{\mathrm{d} P}{\mathrm{~d} t}=(-1+a) P+c-Q, a<1 \\
& \frac{\mathrm{d} Q}{\mathrm{~d} t}=P-\left(d+b Q^{2}\right) Q, b \geq 0
\end{aligned}
$$

This is under the assumption that speeds of adjustment $\alpha=\beta=1$. The demand function is given as $F=(-1+a) P+c$ while the cost function is given as $\left(d+b Q^{2}\right) Q$ where the demand curve $Q=(-1+a) P+c$ is linear and with negative slope. The supply curve $P=b Q^{3}+d Q$ is nonlinear and has both linear and cubic contribution. According to the economic theory, the price elasticity of demand is defined as the percentage change in quantity demanded divided by the percentage change in price $\left(E=-\frac{d Q / Q}{d P / P}\right)$. The elasticity of demand is proportional to the negative slope $(-d Q / d P=1-a)$ 
of the demand curve. Meanwhile, it is convenient for discussion to add a term $\mathrm{c}$ which denotes the shift of the demand curve. If $\mathrm{c}$ is greater than zero, the demand curve shifts to the right. Conversely, the demand curve shifts to the left.

In the theory of conventional economy, demand curve is a downward straight line and supply curve is an upward straight line. Here certain part of the supply curve has negative slope because of the nonlinear cost function as shown in Fig.1. The downward supply curve with respect to $Q$ originates from the excess supply and may often occur in industries and agricultural productions when the income effect is significant. Besides mono-stable equilibrium, the nonlinear demand supply model represents more complicated economic scenarios in different parameters. The demand curve and supply curve intersect at three points or an unstable point. The graphs of demand and supply curve shown in Fig.1 show two scenarios where the bi-stability and limit cycle emerge. Negative values of price and quantity in the current
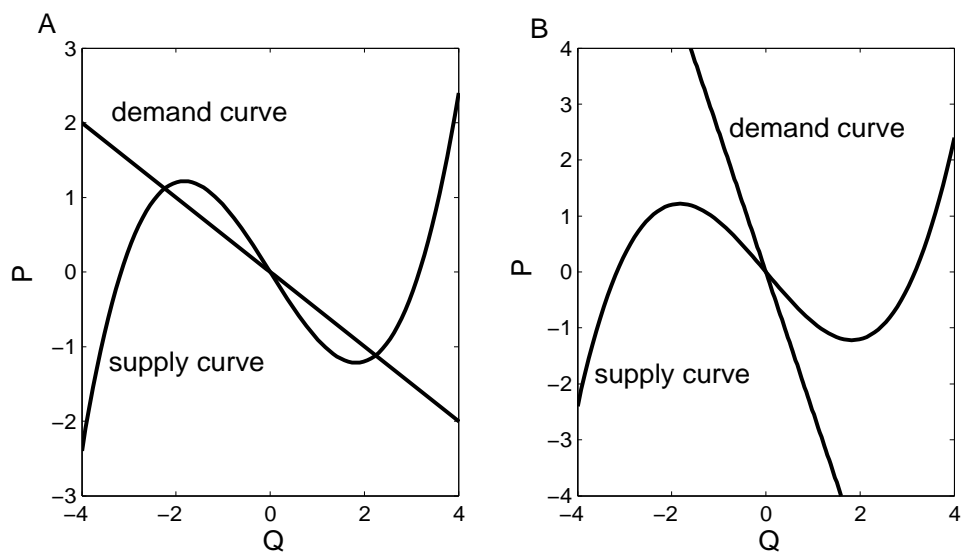

Figure 1: (A)demand curve and supply curve in bistable model (B)demand curve and supply curve in limit cycle model

model is not of our concern here, since they can all be changed to be positive reasonably through parallel shifting simultaneously the supply and demand curves.

\subsection{The Bifurcation of Demand and Supply Model}

In order to explore the nonlinear dynamics under the changes of different parameter, we need to analyze the bifurcation of demand and supply model for different conditions. When we fix another parameters $b=$ 
$0.1, c=0, d=-1$ except for parameter $a$, we can obtain the phase diagram with a super-critical pitchfork bifurcation. It is also a Hopf bifurcation shown in the Fig.2(A). The solid lines denote stable fixed points and the dash lines denote unstable fixed point. We can see two stable states and a unstable state merge to an unstable point(limit cycle) at the critical point. Likewise, when we change the parallel shift parameter $c$ at parameter $a=-1(a<0), b=0.1, d=-1$, we can obtain the phase diagram with saddle-node bifurcation shown in Fig.2(B). The model produces the bistability or a mono-stability with different parameters of $c$. Further, if we change the parallel shift parameter $c$ at the other parameters $a=0.6(0<a<1), b=0.1, d=-1$. the phase diagram is also with a Hopf bifurcation. The model emerges a stable or an unstable point. The two states containing a stable point and a limit cycle can transform to each other at the bifurcation point. This is illustrated in Fig.2(C).
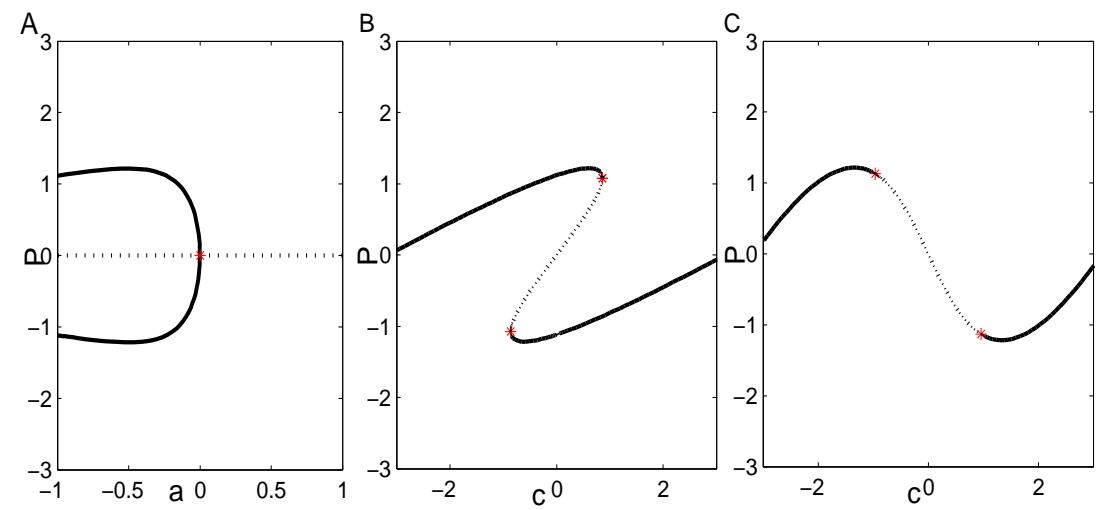

Figure 2: The phase diagram for changing the parameter a or c (A) $\alpha=1, \beta=1, b=$ $0.1, c=0, d=-1 ;(\mathrm{B}) \alpha=1, \beta=1, a=-1, b=0.1, d=-1 ;(\mathrm{C}) \alpha=1, \beta=1, a=0.6, b=$ $0.1, d=-1 ;$ (Solid line : stable; Dash line: unstable)

\section{Results and Discussions}

\section{Landscape and Flux of Conventional Mono-stable Equilibrium Economy}

In the conventional economic theory, both supply curve and demand curve are the straight lines. The two curves possess an intersection on the simple single market model, which is called equilibrium of the market. The price at 
the intersection is the equilibrium price and the quantity of the intersection is called the equilibrium quantity $[27,28]$. We can first study this case from our landscape and flux theory by setting $b=0$ and $d>0$ in our nonlinear supply-demand model. The Fig.3 shows the underlying landscape of the linear supply and demand model. As we can see only one basin of attraction emerges corresponding to the conventional equilibrium state. The basin has a funneled shape. The global stability and robustness can be quantified by the global stability ratio of the gap between the lowest potential and the average of the potential versus the fluctuations in potential represented by the standard fluctuations or square root of the second order variances. This dimensionless global stability ratio represents the slope against the roughness of the underling landscape and quantifies the degree of funnelness, and therefore the global stability.

We can see as the slope parameter $a$ for the demand curve changes, the global stability ratio changes, reflecting the differences in the global stability. With the increasing of slope parameter $a$, the elasticity of demand decreases. For the higher elastic goods, certain changes of quantity demanded bring to small changes in price. Therefore, the equilibrium of the market is more stable. Conversely, when the elasticity of goods is lower, certain changes of quantity demanded can cause significant changes in the price of goods, leading to less stable market.

Once the quantity demanded of the market generates certain fluctuation$\mathrm{s}$, price can present considerable variations. The market becomes unstable in this situation. The conclusion is consistent with the results shown in the Fig.3(C). When the slope parameter $a$ increases, the global stability ratio decreases in the figure. As shown in the Fig.3(D), we also see how the global stability quantified by the robustness ratio changes when the parallel shifts in demand through $c$ is realized. But we find the global stability is almost unchanged with respect to the changes of parallel shift parameters $c$ under the circumstance of the fixed elasticity of demand. The scenario states that the global stability is closely related to the elasticity of the demand, but rarely associates with the parallel shift of the demand. When the quantity demanded has the fluctuations, the range of the price fluctuation is not changed with the parallel shift of demand curve. So the parallel shift parameter can not have impact on global stability of the conventional demand supply model.

We can also see in Fig.3(A)(B), the flux around the basin funnel of the equilibrium state. The dynamics of the underlying economic dynamics can be viewed in the landscape and flux theory as spiraling down to the funnel due 
to the presence of the flux rather than the conventional view of the gradient alone down to the funnel without flux. Furthermore, we have investigated the energy dissipation in the conventional economic model. As the demand curve changes according to $a$ and $c$, the energy dissipation changes. As shown in the Fig.3(E)(F), the more stable funnel requires less energy dissipation to reach the equilibrium. We can see, even in the conventional economic model of supply and demand, the landscape and flux provide new insights towards the identification of equilibrium state as basins of attraction on the landscape with higher probability, global stability quantification through the funnel landscape topography, spiraling dynamics towards the equilibrium state, stability change and the energy dissipation associated with respect to the shift of demand curve.
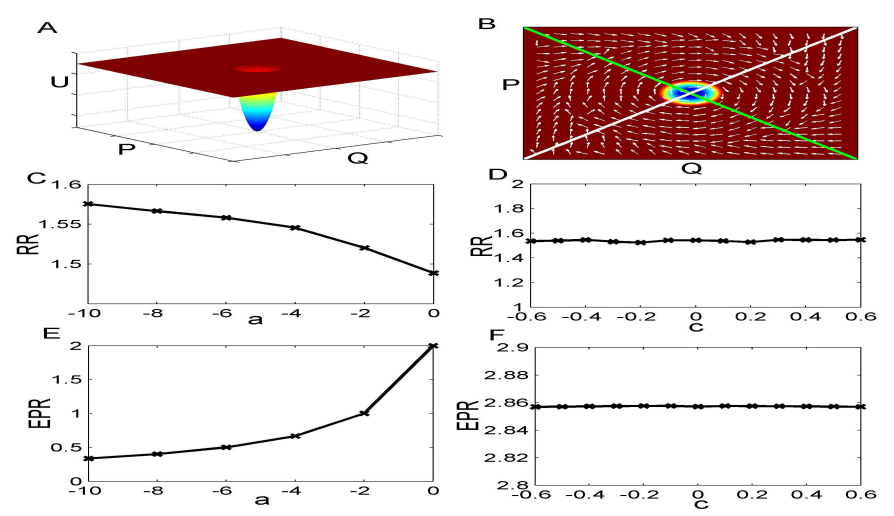

Figure 3: (A)The three dimensional landscape of classical model;(B)The two dimensional landscape of classical model; (C)The Global Stability Ratio versus slope parameter a;(D)The Global Stability Ratio versus shift parameter c; (E)The entropy production rate versus slope parameter a; $(\mathrm{F})$ The entropy production rate versus shift parameter c;

\section{Landscape and Flux of Bistable Non-equilibrium Open Economy}

In the landscape and flux theory of economy, we investigate a nonlinear stochastic dynamic equation underlying supply and demand model. For the new demand supply model, $b \neq 0$. The different slopes $a$ in demand curve can lead to different dynamical behavior. When $0<a<1$, demand curve is a downward sloped line. The intersection between the supply and demand curve gives a unique unstable point. A limit cycle emerges surrounding the unstable point. On the other hand, the demand curve and supply curve can 
have three intersections in the demand slope regime of $a<0$. two of them are stable equilibrium point and the rest is unstable saddle point.
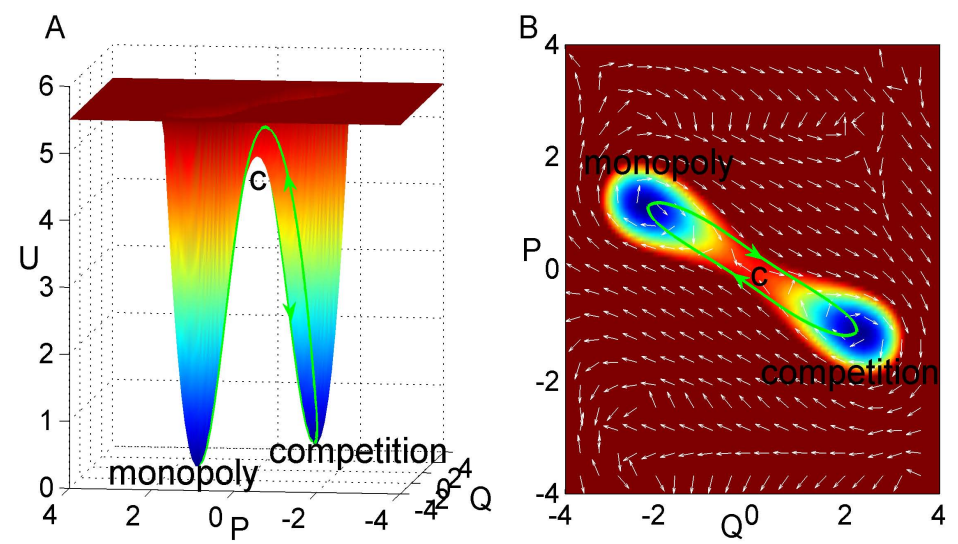

Figure 4: The landscape and dominant path of bistable model
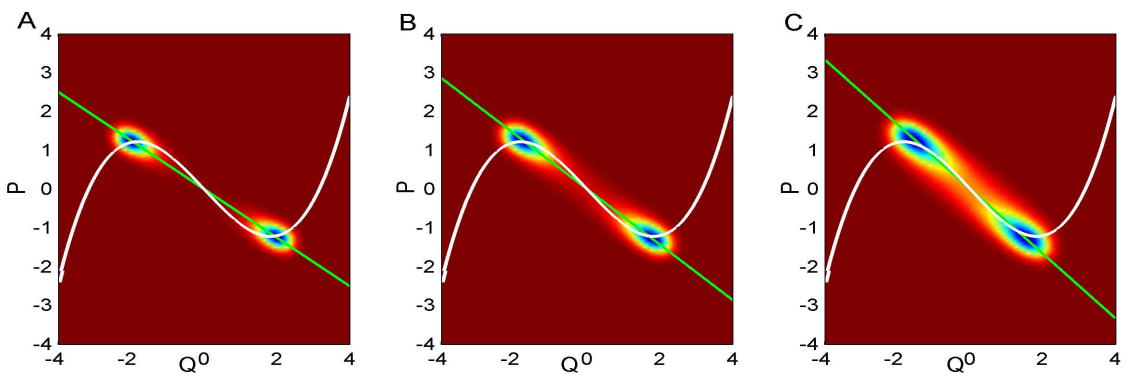

Figure 5: Demand curve, supply curve and the landscapes with different parameter a in bistable model. (A) $\mathrm{a}=-0.6,(\mathrm{~B}) \mathrm{a}=-0.4,(\mathrm{C}) \mathrm{a}=-0.2$

\subsection{Landscape as well as Dominant Paths and Flux of Bistable Economy}

Let us first study the bi-stable equilibrium case. In this case, the potential landscape in terms of steady state probability distribution $(-\ln \mathrm{P})$ is quantified in Fig.4. We find that the three dimensional landscape of probability have two clear maximum region in the variable space of the quantity and the price corresponding to the two basins of attractions on the landscape, which are the two economic stable states. In one of the stable states, the production is cheaper in price with a larger number of quantities. The 
nature of this state is the same as the goods market with the complete competition. Customers will buy the commodity with a low price because of the rich supply products from the competitive conduct of the free market. On the other hand, the production is higher in price with the quantities for the other stable states. The nature of this state resembles the commodity from only one company (a monopoly) or a few (an oligopoly). Customers will still buy some of the goods even at a higher price because of the formation of monopoly or oligopoly price. The depths of the basins of attraction gives the probability or the weights of each state [29].

The Fig.4(B) shows the two dimensional landscape and dominant paths. The optimal kinetic paths are calculated by the path integral method. We can find the dominant paths from a stable state to the other are irreversible. In other words, the paths are different from the equilibrium system where the path from a stable state to the other is completely the same as the reverse path. This is because of the emergence of the curl flux. The system breaks the detail balance away from equilibrium. The curl flux gives rise to the irreversibility of the optimal economic paths. The dominant paths do not pass through the saddle point(c) between landscape basins of attractions(a and $b$ ).

The Fig. 5 shows the two dimensional landscape of bistability in different demand slopes $a$. The white line and green line denote the demand curve and supply curve. We find that the landscape has two minimal(blue region) states which correspond to the two intersections of demand and supply curve. With the increasing of the demand slope $a$, the two steady basin states change gradually. The differences of the potential between the saddle point and stable point become smaller.

\subsection{Global Stability of Bistable Economy}

Through solving the Hamiltonian-Jacobian equation, we can obtain the Lyapunov function of the model. The Lyapunov function of the bistable model are shown in Fig.6. The value of the function is greater than zero in arbitrary area. The value of blue region is less than the red region. The two blue basins denote the two minimum. According to the nature of the Lyapunov function, the temporal evolution monotonically goes downward. The global stability is thus guaranteed. Furthermore, the barrier between the two basins can quantify the degree of difficulty of switching from one basin to another. The higher the barrier, the harder it is to escape from one 
basin to another and therefore the more stable it is. This provides another measure of global stability.
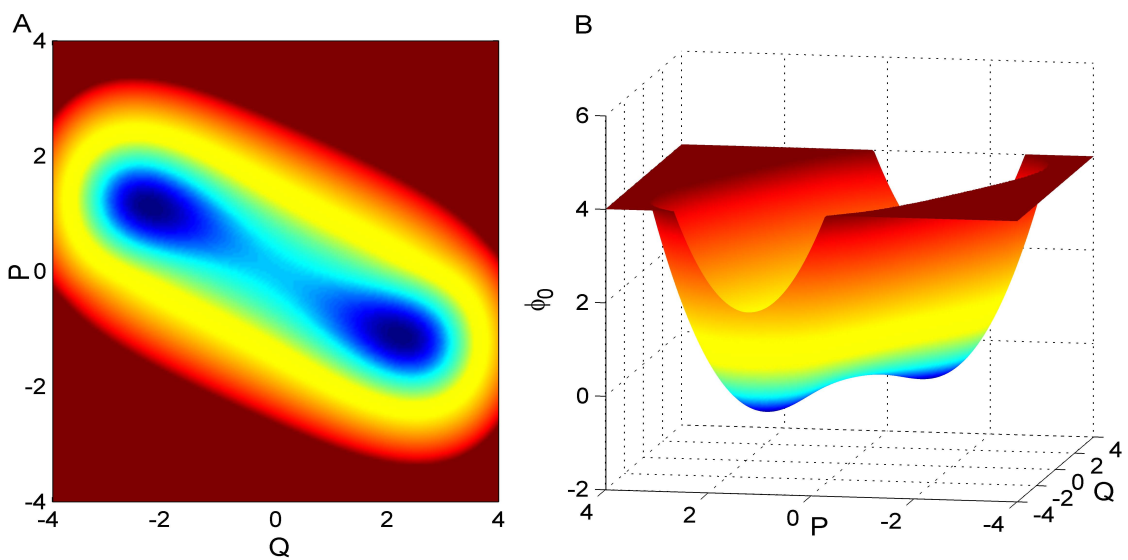

Figure 6: The Lyapunov potential function of the bistable economic model

\subsection{Dissipation, Kinetics and Landscape Topography of Bistable Economy}

The Fig.7(A) shows the entropy production rate as a function of the slope of the demand curve, $a$. We find that entropy production rate increases with the increase of $a$. The demand slope parameter $a$ is closely associated with the elasticity of the demand.Therefore, we can see the energy consumption or dissipation increases with the decrease of the elasticity of demand for production. The rigidity in demand costs more. In Fig.7(B), we show the barrier height along the dominant paths between the basins of attraction with respect to different values of demand slopes $a$. Since the system is symmetric, so we only draw one of the barrier heights along the dominant path between two basins of attraction. We found the barrier height decreases with the increase of the demand slope $a$.

In order to demonstrate the rational of the connection between the barrier height and the demand slope parameter $a$ in goods market, we start with the initial state as monopoly state in the bistable supply and demand model. When demand slope parameter $a$ increases or the elasticity of demand decreases, the total revenue $\mathrm{P}^{*} \mathrm{Q}$ increases. Part of sellers can join the sales of the commodity. The monopoly state then becomes unstable. The barrier height can then decrease, and the monopoly state transforms into the competition state easily. Secondly, if the initial state is in competition state 
and the elasticity of the demand decreases, then both the equilibrium price and equilibrium quantity decrease. The total revenue will go down. Part of sellers may abandon the sales of the goods. The competition state becomes unstable. So the increasing of the demand slope parameters $a$ can decrease the barrier height or the stability of a state. The more (less) elasticity in demand will favors more (less) stable economy.

In Fig.7(C), we calculated the mean first passage time from a state to $\mathrm{b}$ state. On the basis of the same reasoning, we only calculated one of the two results of forward and backward transitions. We found that the mean first passage time from one to another becomes shorter with the increase of the demand slope $a$. The result is consistent with Fig.7(B). When the barrier height along the landscape becomes lower, the mean first passage time also decreases. Consequently, the transition from a stable state to the other will be easier. The Fig.7(D) plots the integral of the flux $J_{s s}$ along the closed loop of the dominant forward and backward paths with respect to demand slope a. The integration over flux gives a scalar measure on the effect of the curl flux. We found the integrated flux along dominant path loop increases with the increase of the demand slope a.

Fig.8(A) shows the change of the entropy production rate with respect to the integral of the flux $J_{s s}$ along the closed loop of the dominant forward and backward paths. we find the entropy production rate increases with the rise of the integral of flux. The more flux will produce more heat dissipation for the nonequilibrium economic systems. Fig.8(B)shows the change of the mean first passage time with respect to the barrier height between saddle $\mathrm{c}$ and point $\mathrm{a}$. we can see the time becomes longer with the increasing of barrier height. Obviously, the time is correlated to the barrier height in the bistable model.

\subsection{Global Sensitivity Analysis for the Key Parameters Factors Determining the Stability of Bistable Economy}

As we have seen, landscape barrier and MFPT between two basins of attraction represent the global nature of the economic system, they can be used as the global stability measures to ask the question which parameters in the economic system can change the global stability and function significantly. Those changes significantly are the ones crucial for determining the stability and dynamics of the economy. They can be identified through global sensitivity analysis. In Fig.9, We show some parameters which influence the barrier height along the dominant paths. We can see that the demand 

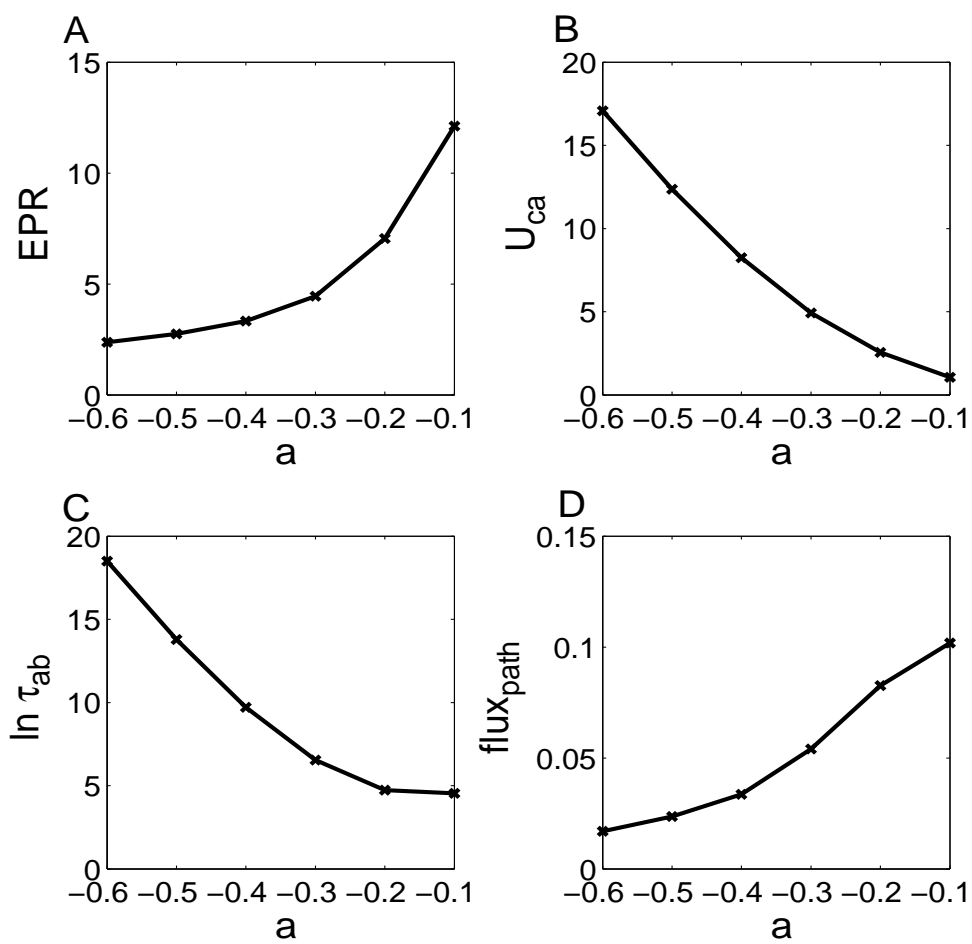

Figure 7: (A)Entropy production rate with different diffusion coefficient;(B)Barrier height between point $\mathrm{c}$ and point a; (C)Mean first passage time with different diffusion coefficient; (D)The integration of $J_{s s}$ along the closed loop of the dominant path with different diffusion coefficient;

slope parameter $a$ has more significant effects on the barrier heights along the dominant paths. Our method is general and can predict the influence of parameters on the global stability and robustness of the stochastic economic system. In this way, the "hot" or key structure elements determining the underlying economic dynamics can be identified. Based on the results, one maybe able to establish better policy to influence the economy globally. 

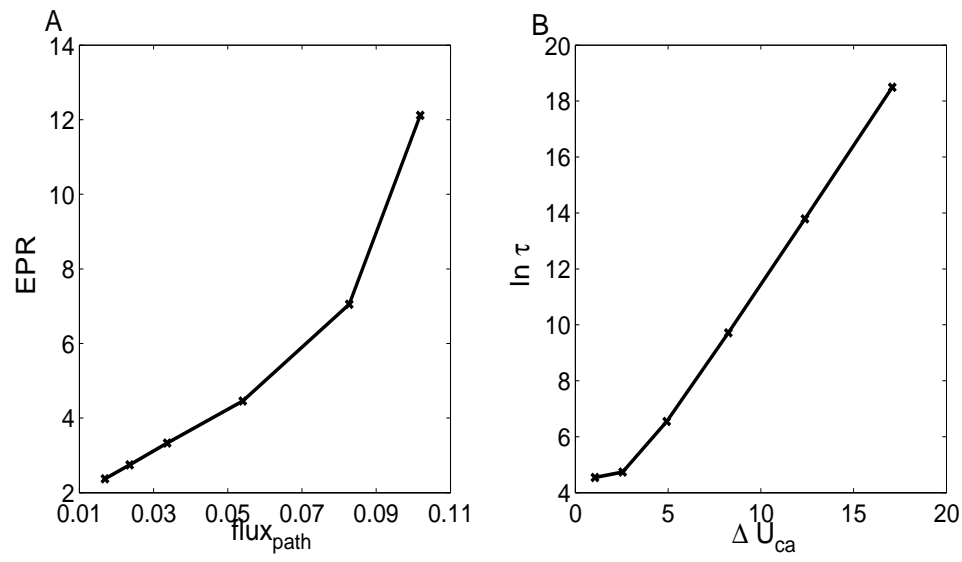

Figure 8: (A)Entropy Production Rate with different integrated flux;(B)Mean first passage time with different barrier height between $\mathrm{c}$ and point $\mathrm{a}$;

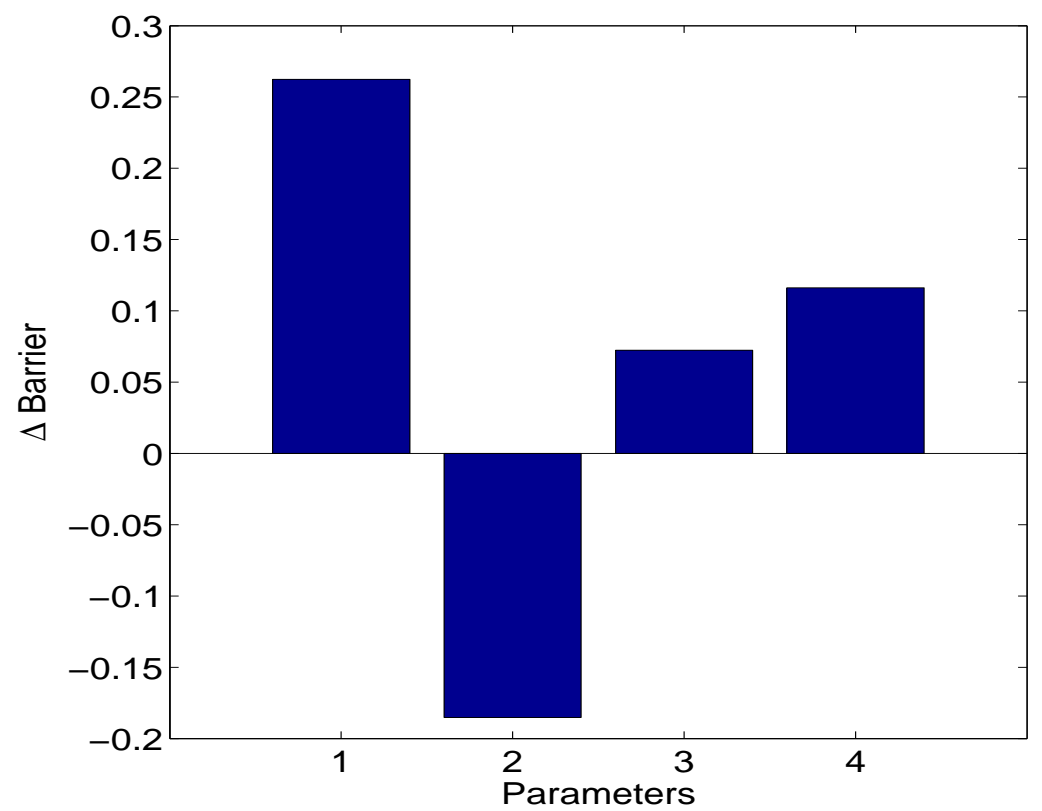

Figure 9: Sensitivity analysis for bistable economy. (1: a; 2 : b; $3: \alpha ; 4: \beta)$

\section{Landscape and Flux of Limit Cycle Non-equilibrium Open E- conomy}

5.1. Driving Force in Terms of Landscape and Flux for Limit Cycle Oscillating Economy

Further, we will explore the scenario of a cycle limit for the supply demand model. According to the same method, we quantify the underlying 
landscape and found higher probability along the limit cycle trajectory. The landscapes and limit cycle trajectory are shown in Fig.10 . The landscape has an irregular Mexican hat shape with a center island in the middle and an oscillation ring valley surrounding the center island separated from the outer higher plateaus. Outside the oscillation ring valley, the dynamics is determined mainly by the gradient of the landscape, attracting the states down to the oscillation ring. On the oscillation ring valley, we can see two local basins with higher probabilities. These two basins along the oscillation ring valley correspond to the two stable states in the bi-stability regime. The higher probability on the oscillation path implies the longer duration. Therefore this implies the oscillation along the ring valley is not uniform in speed at different locations. Here we can see there is no bi-stability but the inhomogeneous oscillations along the valley paths. The local basins and barriers on the oscillation path tend to drive the system down to the local basins on the ring valley according to their gradients. To keep the oscillation running, there has to be another driving force. This is provided by the curl flux. The oscillation dynamics on the ring valley of the Mexican hat shape landscape is then determined by both the flux driving the cycle motion and local basins and barriers dragging the cycle dynamics. As we can see, both the landscape and flux are critical for coherent limit cycle oscillation in this supply and demand dynamics. In this case, the goods market presents periodic behaviour around the unstable point and do not have a stable equilibrium state.

A

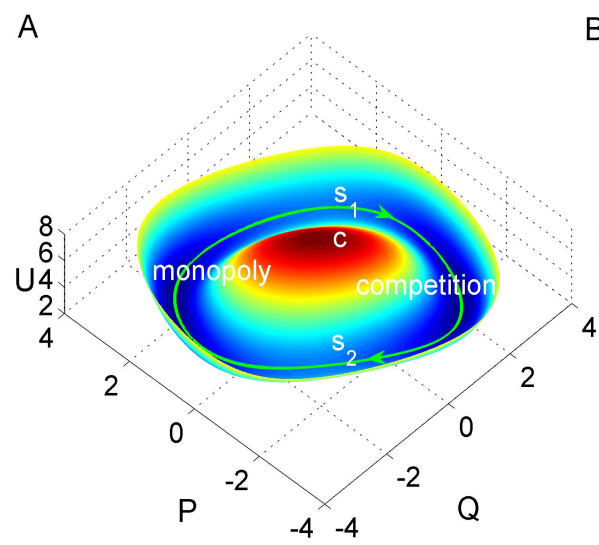

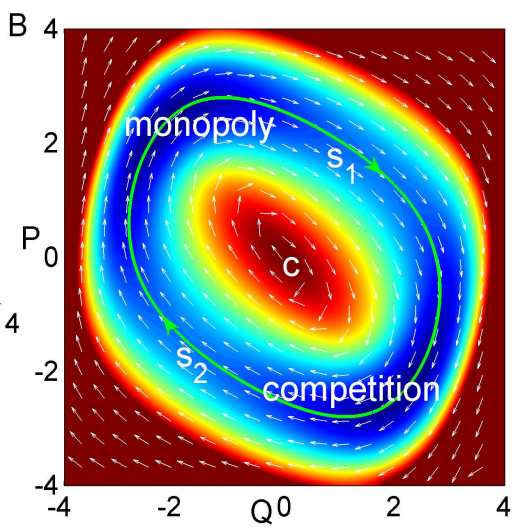

Figure 10: The landscape and limit cycle

Fig.11 shows the two dimension landscape of limit cycle at different demand slope $a$. We find that the landscapes have circular ring and two clear 
minimal(blue) region. With the increasing of the demand slope $a$, The circular ring becomes larger and larger.
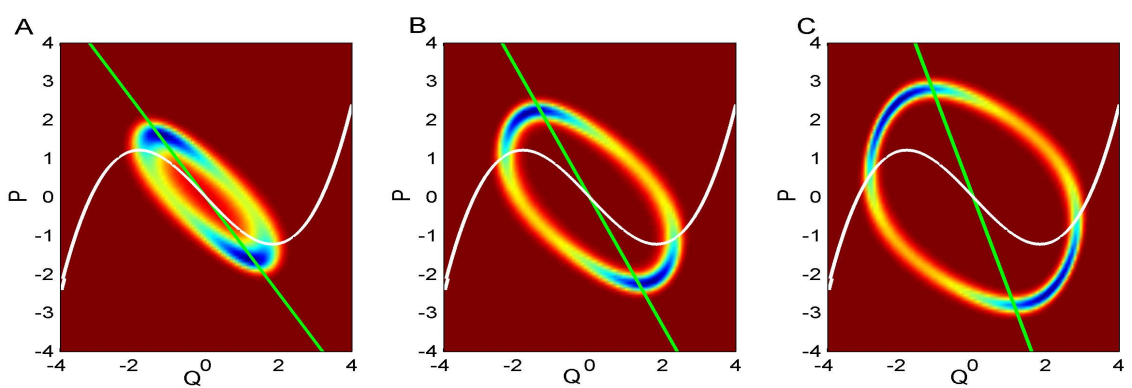

Figure 11: Demand curve, supply curve and the landscapes with different parameter a in limit cycle model. (A) $\mathrm{a}=0.2$, (B) $\mathrm{a}=0.4,(\mathrm{C}) \mathrm{a}=0.6$

\subsection{Lyapunov Function and Global Stability for Limit Cycle Economy}

Fig.12 shows the Lyapunov function of the limit cycle model. The value of blue region is less than the red region. The blue ring valley is located at the bottom of the Lyapunov function. According to the nature of the Lyapunov function, the temporal evolution monotonically decreases. At the bottom of the ring valley, the Lyapunov function becomes constant. In fact, this is a signature of Lyapunov function for continuous attractors such as limit cycle. If Lyapunov function is not a constant at the ring valley, then when going around the ring, there is always a trend to increase. But Lyapunov function always decreases. This creates a contradiction. Therefore, at the bottom ring valley, Lyapounov function is constant. The global stability can also be quantified as the barrier height between the top of the center island (or top of the Mexican hat) and the ring valley. When this barrier is high, then global stable oscillation is guaranteed. On the other hand, the lower barrier will make oscillation less stable and eventually disappear.

\subsection{Market elasticity, Flux, Dissipation, Kinetics and Landscape Topography for Limit Cycle Economy}

Fig.13(A) shows the entropy production rate as function of demand slope parameter $a$. We found that entropy production rate increases with the increase of demand slope $a$. Therefore, we can see that the energy consumption or dissipation increases with the decrease of the elasticity of demand of production. In Fig.13(B), we show the barrier height between the state 

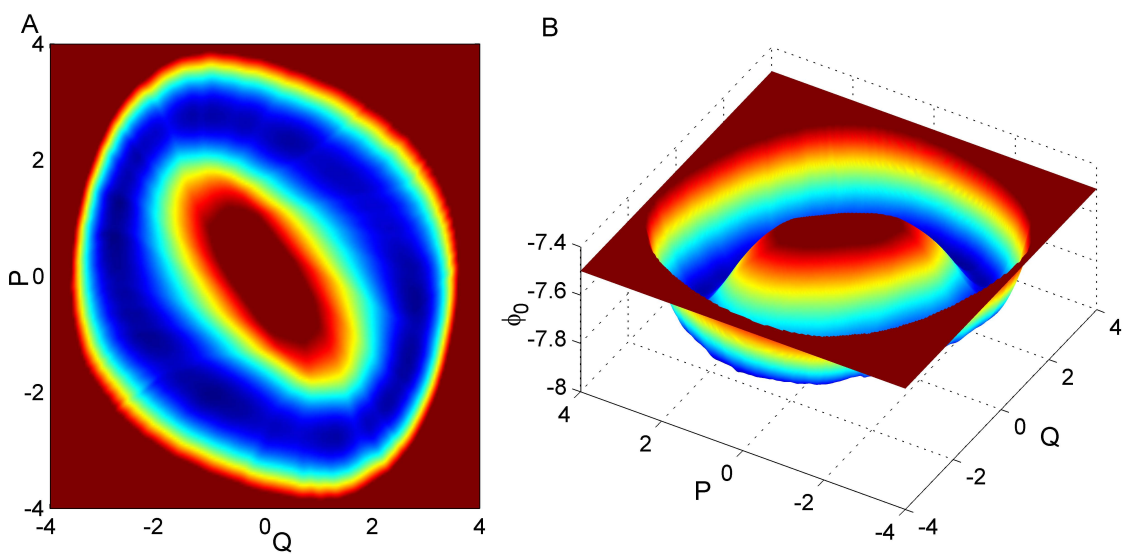

Figure 12: The Lyapunov potential function of the limit cycle economic model

of largest probability along the oscillation path and the center island with respect to demand slope parameter $a$. We found the barrier height of the center island relative to the oscillation ring becomes bigger with the increase of demand slope $a$. In Fig.13(C), we calculated the mean first passage time from a high probability state along the oscillation path to the center island. We can see the mean first passage time also increases with the increase of demand slope $a$. This result is consistent with Fig.13(B). The transition from a high probability state along the path to the center can hardly be realized. The Fig.13(D) shows the integral of flux $J_{s s}$ along the limit cycle with respect to the demand slope parameter $a$. We found the integrated flux increases with the increase of the demand slope parameter $a$ or the decrease of the elasticity of demand of production. Meanwhile, the energy dissipation also increases. The more flux will generate more heat dissipation for the nonequilibrium economic system.

Fig.14(A) shows the change of the entropy production rate with respec$\mathrm{t}$ to the integral of the flux $J_{s s}$ along the closed limit cycle. we find the entropy production rate goes up with the increasing of the integral of flux. Likewise, we can find the same fact that the more flux will produce more heat dissipation . Fig.14(B)shows the change of the mean first passage time with respect to the barrier height between center $\mathrm{c}$ and point a. we can see the time become longer with the increasing of barrier height. Obviously, the time is also closely related to the barrier height. In other words, the higher barrier height leads to the more stable limit cycle. 

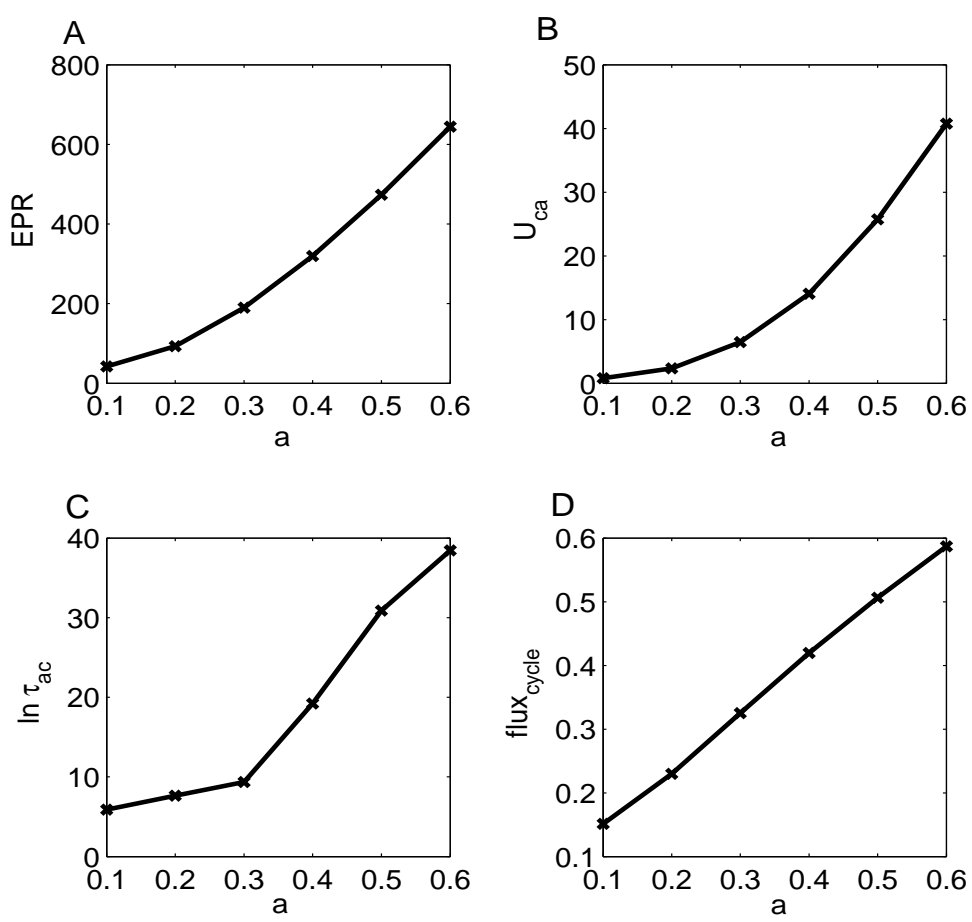

Figure 13: (A)Entropy production rate with different diffusion coefficient;(B)Barrier height between point $\mathrm{c}$ and point a; (C)Mean first passage time with different diffusion coefficient; (D)The integration of $J_{s s}$ along the limit cycle with different diffusion coefficient;

\subsection{Coherence, Period, Market Elasticity and Flux of Limit Cycle Economy}

In Fig.15(A)(C), we calculated the coherence with different demand slope $a$ and different integral of flux. We find the coherence increases with the increase of demand slope $a$. This means the coherence of the oscillations increases when the elasticity of demand decreases. Likewise, the coherence of the oscillations also strengthens with the increase of integral of flux. In Fig.15(B)(D), we calculated the period of the system with different demand slope $a$ and different integral of flux. We can see the period becomes shorter with the increase of demand slope $a$. And the period becomes also shorter with the increase of integral of flux. 

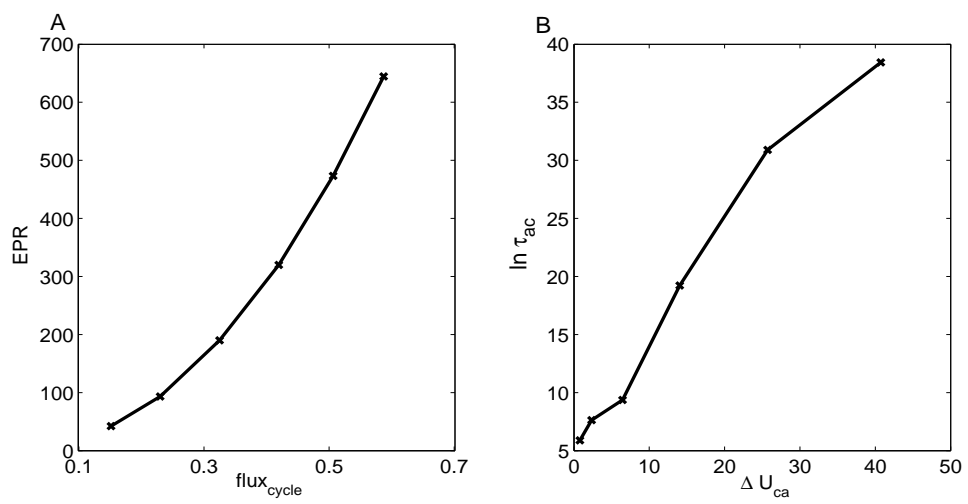

Figure 14: (A)Entropy Production Rate with different integrated flux;(B)Mean first passage time with different barrier height between $\mathrm{c}$ and point a;

\subsection{Origin of Limit Cycle Economy}

When the elasticity of demand or the slope parameter $a$ reaches a threshold, supply and demand can't realize the two stable states. With the increasing of slope parameter $a$, the elasticity of demand decreases. If goods market is in monopoly state, the demand quantity for the same price increases more than in the bistable states. The lack of the supply quantity leads to the rise of the goods price. Meanwhile, the effective demand quantity provisionally decreases and the effective supply quantity provisionally increases. the market constitutes a transient steady stability. With the slowly increasing of the supply quantity, the goods price will decrease. The transient monopoly state is broken then to reach the competition state. Similarly, if goods market starts with the competition state, the demand quantity for the same price greatly decreases with the elasticity of demand decreases. The goods price becomes lower. Meanwhile, the effective demand quantity will rise up and the effective supply quantity provisionally decreases. The market forms a transient steady stability. Then the transient steady state price and quantity are broken again. The market goes back to monopoly state. The transient stable competition state and monopoly state appear alternately, So the goods market shapes a period oscillation.

\subsection{Relationship between Elasticity of Demand and Stability}

In the above, we mention the stability increases with the increasing of the elasticity of demand for bistability and monostability. But we obtain opposite trend for limit cycle economy. It is clear RR for single basin and barrier for 

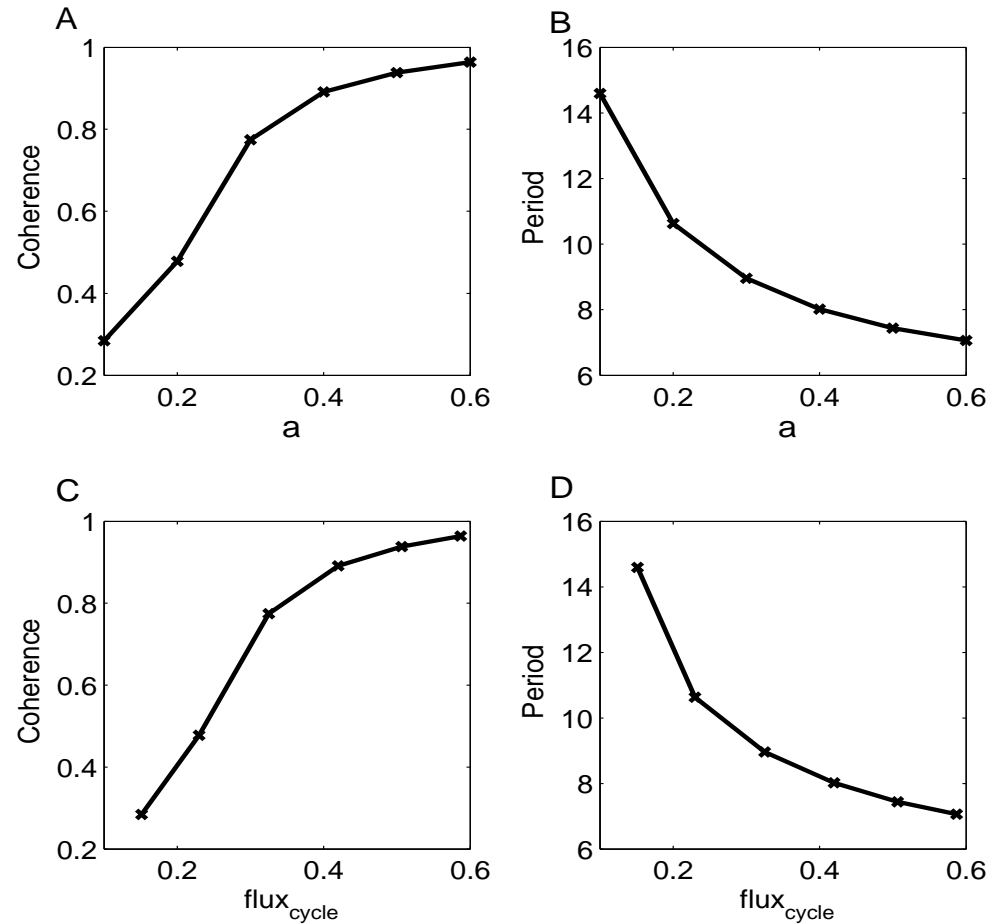

Figure 15: (A) Coherence with different parameters a . (B) The period with different parameters a. (C) Coherence with different integration of $J_{s s}$ along loop cycle . (D) The period with different integration of $J_{s s}$ along loop cycle

bistable basins increase as the elasticity of demand increases. That means more elastic market favors higher stability since the market is more easily adapted to the change. More rigid economy would not be easily adapted to the changes and therefore less stable towards the perturbations. But why in the limit cycle things seem opposite? We think about the limit cycle as the flow between two states (competition and monopoly), then the higher (lower) stability of the flow should be the barriers between these two states along the oscillation path indicates lower (higher). In other words, in order to maintain the higher (lower) stability of the limit cycle flow, the two states (competition and monopoly) should be less (more) stable. Therefore, this is consistent with the monostability and bistability discussion. The higher elasticity favors the stability of point attractors and disfavors the flow (limit cycle as continuous attractor). The lower elasticity favors the flow (limit cycle) but disfavors the 
point attractors. On the other hand, with the decreasing of the elasticity of demand, the open economic system transforms the dominant driving force from spontaneous equilibrium into the dominant flux from period oscillation. So the system is transformed from bistability to limit cycle. The main effect of gradient force of potential guarantees the stability of bistable system, but the dominant impact of the flux insures that the limit cycle system is more stable. Therefore, the elasticity of demand has the different effect on the stability of the bistability and limit cycle.

\subsection{Global Sensitivity Analysis for Identifying Key Elements for Limit Cycle Economy}

We also performed the global sensitivity analysis of underlying parameters. In Fig.16, we show some parameters that influence more on the barrier height between center island and oscillation paths. We found that the demand slope parameter $a$ has more influence on barrier height. This result further explains the importance and the influence of the elasticity of demand.

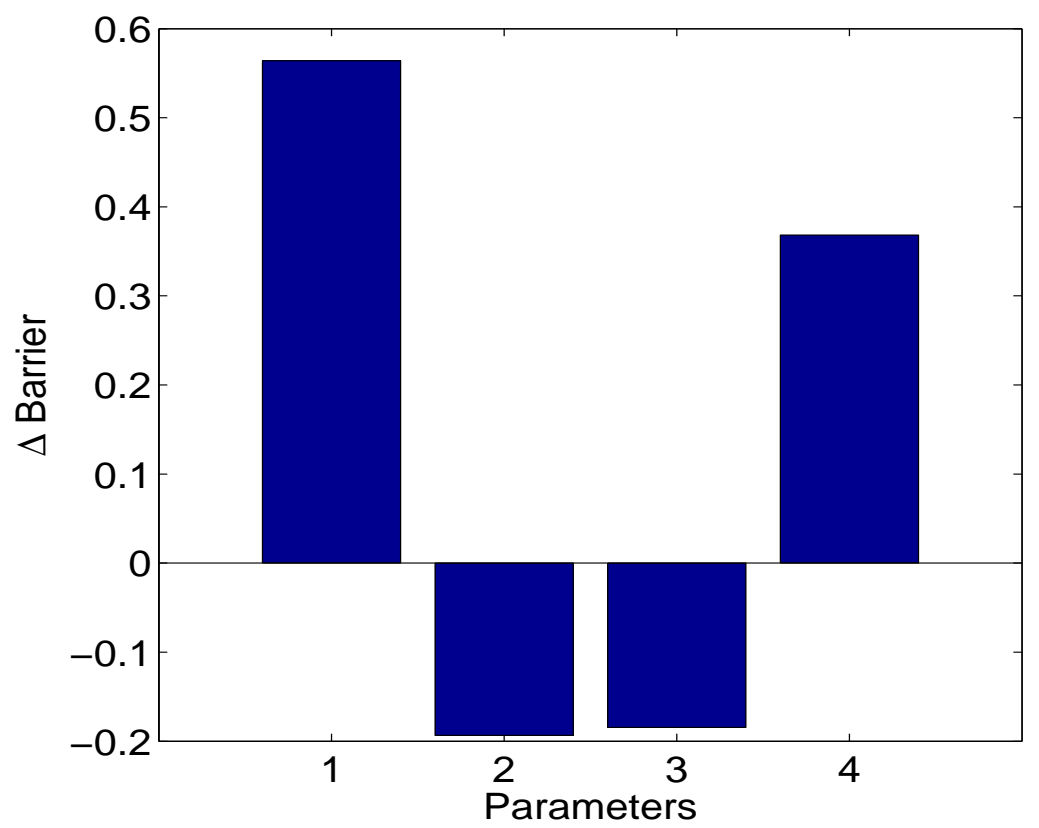

Figure 16: Sensitivity analysis for limit cycle economy. (1: a; $2: \mathrm{b} ; 3: \alpha ; 4: \beta)$ 


\title{
6. Landscape and Flux of Bistable and Limit Cycle Non-equilibrium Open Economy with Parallel Shift in Demand or Supply
}

\author{
6.1. Landscape and Flux of Bistable Economy Under Parallel Shift in De- \\ mand or Supply
}

In the study of supply and demand model of conventional economy, researchers often explore the shift of the demand curve to understand the dynamics of the market. The shift of the demand curve denotes the change of the relationship between the quantity demanded and price when certain economic conditions happen to change. For example, the income increases or the substitute appears. Therefore, we also explore and quantify the change of the landscapes when the demand curve varies with parallel shift parameter $c$ in Fig.17. We found the basin of attraction of state "b" (competition state) becomes deeper and more prominent relative to the state "a" (monopoly state) on the landscape when the demand curve shifts to the right slightly. Once the demand curve shifts to the right over the certain value, the basin of attraction of state "a" will disappear and only state "b" survives on the landscape. In other words, the probability of the state "b" increases when the demand increases. Once the increase in demand exceeds certain value, the probability of the state "a" will be close to zero. At this time, we believe the state "a" and "b" of the system evolve to only one state. In other words, the system preserves only one stable state. The model returns to the conventional supply and demand model where only one equilibrium state typically appears. Furthermore, when the decrease in demand exceeds a certain value or the demand curve shifts to the left, the probability of state "b" will decrease and the state will gradually disappear. We can explain the case in the following figures. When the increase in demand occurs, a large number of sellers would join the production and sales of the goods. The goods market have more competitors and form a more competitive environment. Therefore, the probability of competition state will enlarge in the environment. On the contrary, when the decrease in demand occurs, many sellers break away from the commodity market. The single market would reach less competitive environment, so the probability of monopoly state will increase.

In Fig.18(A), we show the change of the entropy production rate with different shift parameter $\mathrm{c}$ in bistable system. we find the entropy production rate increases first and then decrease with the rising parameter c. This means the entropy production rate has an optimum value without the shift of demand curve. Bistable economy costs more to maintain than single basin 

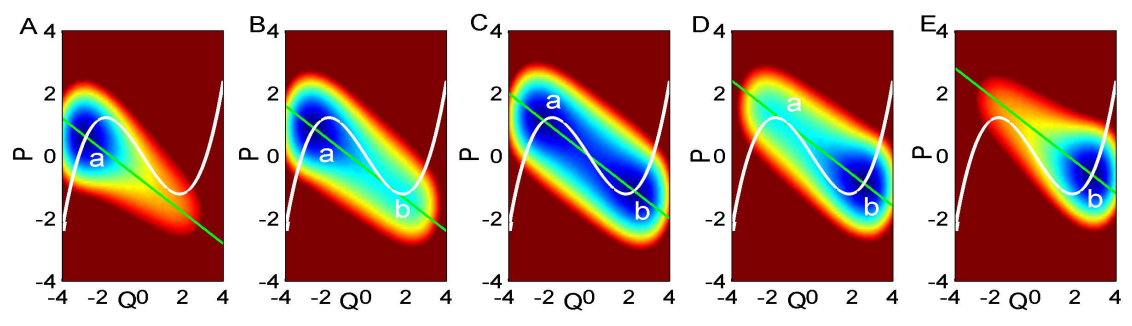

Figure 17: the landscapes of the shift of demand curve on bistable model

economy. In Fig.18(B), we show that the barrier heights with different values of the shift parameter c. we can see the barrier height between the saddle of potential and state a declines with the increase of parameter c. But the barrier height between the saddle and state $b$ rises up with the increase of parameter c. They are identical when parameter c is equal to zero. In other words, the landscape potential of monopoly state becomes smaller than the one of competition state with the left shift of demand curve. Conversely, the landscape potential of monopoly state is larger than the one of competition state with the right shift of demand curve. So the system stays on the monopoly state with higher probability when the demand curve shifts to the left. For the right shift of demand curve, the system stays on the competition state with higher probability. In Fig.18(C), we show the mean first passage time when the shift parameter c changes. we find its tendency is consistent with the barrier height. This also denotes the monopoly state is more stable with the left shift of demand curve. For the right shift of demand curve, the competition state is more stable. And higher barrier height leads to the longer first passage time. In Fig.18(D), we calculated the integral of flux along the loop dominant path with different shift of demand curve. The integral of flux also has a maximum at zero shift. we find the tendency is the same as the entropy production rate. Further, we can see the more flux will produce more energy dissipation.

\subsection{Landscape And Flux of Limit Cycle Economy Under Parallel Shift in Demand or Supply}

Likewise, we can study and quantify the change of the landscapes when the demand curve has parallel shifts in limit cycle model. As shown in Fig.19, we found competition state becomes higher probable and more prominent relative to the monopoly state when the demand curve shifts to the right slightly. Once the demand curve shifts to the right over certain value, the 

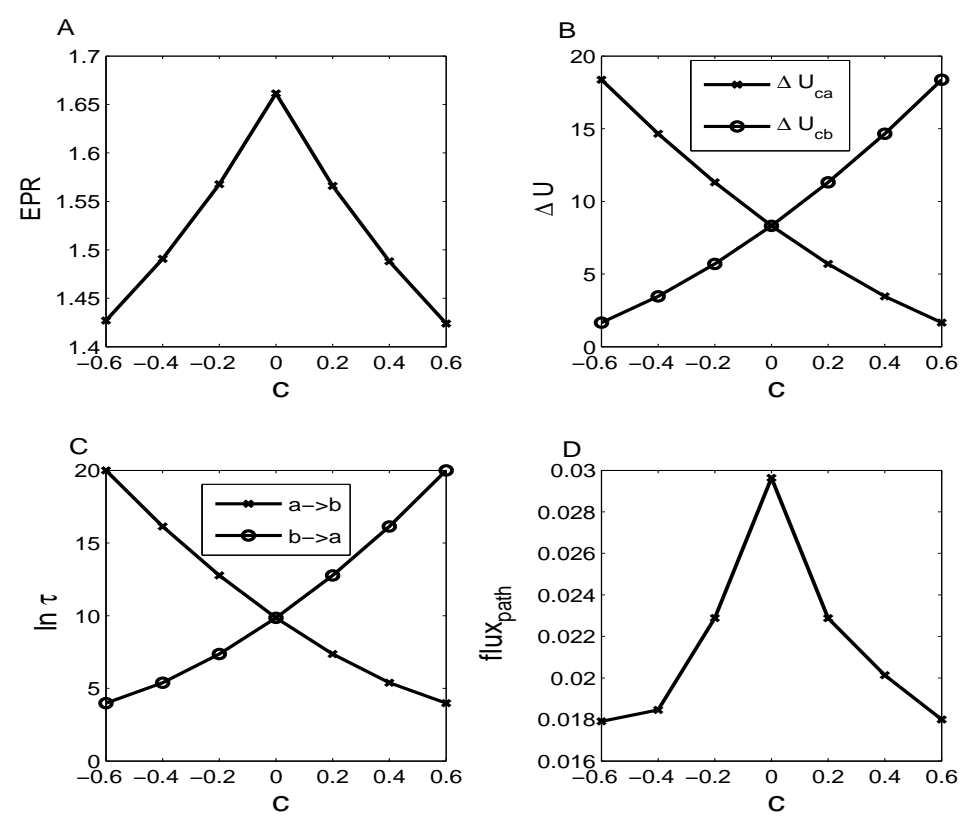

Figure 18: (A)Entropy production rate with different shift parameter c ; (B)Barrier height between point $\mathrm{c}$ and point a with different shift parameter $\mathrm{c}$; (C)Mean first passage time with different shift parameter c ; (D) The integration of $J_{s s}$ along the limit cycle with different parameters $\mathrm{c}$

shape of the Mexican hat will completely change and only state "b" survives on the landscape. In other words, when the change of demand exceeds certain value, the probability of the state "a" will be close to zero. At this time, the limit cycle system evolves to only one stable state. Conversely, when the decline of demand goes beyond certain value when the demand curve shifts to the left, the probability of competition state will decrease until gradually disappeared. We can also explain this scenario with the similar standpoint of bistable model. When the increase in demand occurs, monopoly can not satisfy the demand of commodity. The market brings more competitors. Therefore, the probability of competition state will enlarge in the goods market. On the contrary, when the decrease in demand occurs, many sellers break away from the commodity market. The single market would enter less competitive environment, so the probability of monopoly state will increase.

In Fig.20(A), we show the change of the entropy production rate with different shift parameter $\mathrm{c}$ in limit cycle. The entropy production rate increases 
first and then decreases with the rising parameter c. It has a maximum at zero shift of demand curve. In Fig.20(B), we show the barrier height with different shift parameter c. We can see the barrier height between the center island and "a" or "b" state goes up first then declines with the increase of parameter c. And the two values are almost identical. In other words, the limit cycle is more stable with no parallel shift. In Fig.20(C), we show the mean first passage time when the shift parameter $\mathrm{c}$ changes. we find that its tendency is consistent with the barrier height. This also denotes the limit cycle is more stable with no parallel shift. In Fig.20(D), we calculate the integral of flux along the cycle path with different shifts of demand curve. The integral of flux also has a maximum at zero shift of c. We can see the tendency is the same as the entropy production rate. Therefore, the more flux will produce the more energy dissipation. The limit cycle economy requires more energy consumption to maintain than a single basin economy.
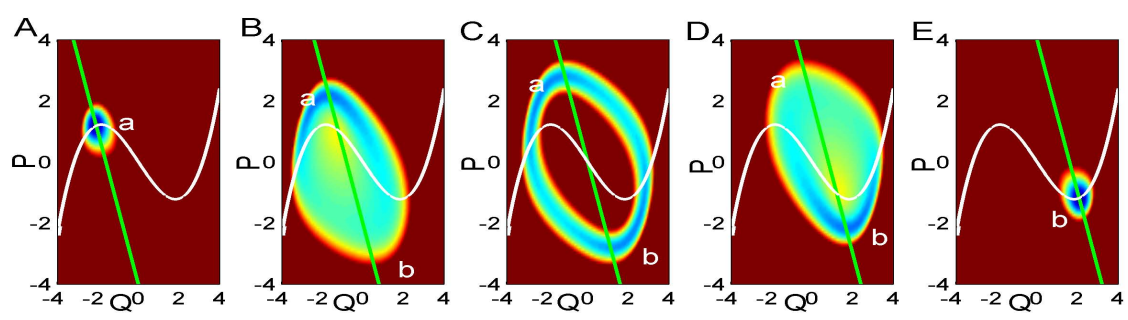

Figure 19: the landscapes of the shift of demand curve on cycle model

\section{Conclusions}

In this work, we developed a landscape and flux theory for describing the general dynamics of non-equilibrium open economy. First, we quantify the states of economy and identify the multi-equilibrium states as the basins of attractions on the underlying landscape. We transform the local description of economic dynamics through individual trajectories to global description in terms of state transitions between basins of attractions. We uncovered the two driving forces of economy, one is from the underlying landscape and the other is from the curl flux which measures the degree of detailed balance breaking. The dynamics of economy thus resembles an electron moving in an electric (landscape gradient) field and magnetic field (curl flux). The explicit identification and quantification of the driving forces like this for eco- 

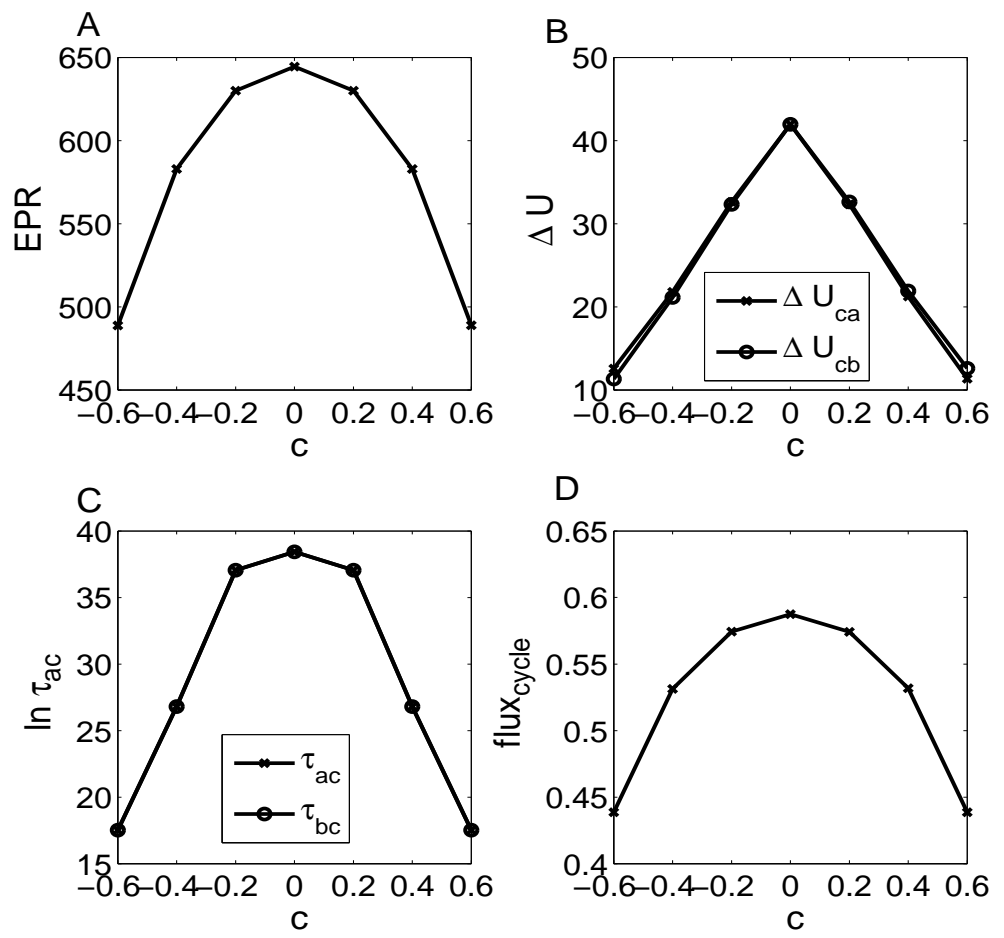

Figure 20: (A)Entropy production rate with different shift parameter c ; (B)Barrier height between center $\mathrm{c}$ and point a or point $\mathrm{c}$ with different shift parameter $\mathrm{c}$; (C)Mean first passage time from point $\mathrm{a}$ or $\mathrm{b}$ to the center $\mathrm{c}$ with different shift parameter $\mathrm{c}$; (D) The integration of $J_{s s}$ along the loop cycle with different parameters shift parameters c

nomic dynamics have not been found before in the conventional equilibrium economics.

In the conventional economy, the supply and demand usually has only one equilibrium point and the trend for changes is described as the shift of the equilibrium. However, the non-equilibrium description of the open economy is missing. By considering nonlinear supply-demand dynamics, we found both bi-stable states of the competition and monopoly/oligopoly and limit cycle oscillations through the competition and monopoly/oligopoly states can emerge.

We uncovered the underlying landscape of bi-stability as the bi-stable basins of attraction with barriers in between. We quantified the optimal paths between the bi-stable basins to uncover the underlying kinetic processes 
from one stable state to another. The paths are irreversible and do not usually pass through the saddle between the basins due to the curl flux. We also quantified the kinetic transition time between the bi-stable basins, which is found to be correlated with the barrier height along the optimal paths. This gives a measure for the global stability of the bi-stable non-equilibrium economy.

We uncovered the underlying irregular Mexican hat shaped landscape of limit cycle dynamics for the supply-demand economy. The dynamics and period/coherence of the limit cycle oscillation are determined by the curl flux and the local barriers along the cycle. Kinetic rates escaping from the cycle, which is found to be correlated with the barrier height between the oscillation ring and the center island, can be used to measure the global stability of the non-equilibrium limit cycle economy.

We quantified the energy cost for non-equilibrium economy and found it is correlated with the flux. For comparison with conventional economy, we shift the demand curve. By shifting the slope of demand curve, we can see how bi-stability transforms to limit cycle and vice versa. By parallel shifting the demand curve, we can also see how monopoly, competition, and bistable monopoly and competition states emerge and transform to one other. We can also see how mono-stable monopoly, limit cycle and mono-stable competition states emerge and transform to one another. The state switching in nonequilibrium economy provides very different pictures for dynamics than the conventional economy of state shifting.

Our landscape and flux theory for non-equilibrium economy is general and can be applied to many economic models for global quantification and mechanism uncovering of the underlying non-equilibrium economic dynamics.

\section{Acknowledgement}

We thanks the support of National Nature Science Foundation of China Grants No. 91430217. JW thanks National Science Foundation NSF-PHY 76066 for support.

\section{References}

[1] Smith Adam(1977). An Inquiry into the Nature and Causes of the Wealth of Nations. University of Chicago Press. 
[2] Walras L (1874)Elements of Economic Politique Pure, 1st Edition, Lausanne:F. Rouge; English translation, William Jaffe, 1954, Elements of Pure Economics, London: Allen and Unwin.

[3] Pareto, Vilfredo, 1909, Manual of Economie Politique, Paris: Girard et Briere.

[4] Rosser JB(2000) From Catastrophe to Chaos: A General Theory of Economic Discontinuities: Mathematics, Microeconomics, Macroeconomics, and Finance, Volume 1, 2nd ed. Boston: Kluwer Academic Publishers

[5] Marshall A.(1890) Principles of Economics. MacMillan,London.

[6] Robinson J.(1933) The Economics of Imperfect competition, Macmillan

[7] Bonanno G.(1987) Monopoly Equilbria and Catastrophe Theory. Australian Economic Papers 26, 197-215

[8] Marx K.(1998) Capital I: The Process of Production of Capital. International Publishers:New York.

[9] Goodwin R.M.(1967) "A Growth Cycle", in C.H. Feinstein, editor, Socialism, Capitalism and Economic Growth. Cambridge: Cambridge University Press

[10] Schumpeter, J. A. (1934) The Theory of Economic Development: An inquiry into profits, capital,credit, interest and the business cycle, Cambridge,MA: Harvard University Press.

[11] Minsky H.(1977) The financial instability hypothesis: an interpretation of Keynes and an alternative to standard theory, Nebraska Journal of Economics and Business

[12] Fisher I.(1933) The debt-deflation theory of great depressions. Econometrica, 1: 337C55.

[13] Keynes J.M.(1936) The General Theory of Employment,Interest and Money, London: Macmillan.

[14] Keen S.(1995) Finance and economic breakdown:modeling Minsky's Financial Instability Hypothesis, Journal of Post Keynesian Economics, 17(4): 607C35. 
[15] Keen S.(2001) Debunking Economics: The Naked Emperor of the Social Sciences. Pluto Press Australia

[16] Beckmann M.,Ryder H.(1969) Simultaneous price and quantity adjustment in a single market. Econometrica 37:470-484.

[17] Pierre N.V.Tu.(1994) Dynamical systems: An Introduction with Applications in Economics and Biology. Springer.

[18] Van Kampen, N. G. (1992) Stochastic Processes in Physics and Chemistry. Elsevier Sience Publishers.

[19] Wang J, Xu L, Wang E (2008) Potential landscape and flux framework of nonequilibrium networks: Robustness, dissipation, and coherence of biochemical oscillations. Proc. Natl. Acad. Sci. USA. 105: 12271-12276.

[20] Feng H, Wang J (2011) Potential and flux decomposition for dynamical systems and nonequilibrium thermodynamics: Curvature, gauge field, and generalized fluctuation-dissipation theorem. J. Chem. Phys. 135: 234511.

[21] Wang J, Zhang K, Wang EK (2010) Kinetic paths, time scale, and underlying landscapes:A path integral framework to study global natures of nonequilibrium systems and networks. J. Chem. Phys. 133, 125103.

[22] Wang J, Zhang K, Xu L, Wang EK,(2011) Quantifying the Waddington landscape and biological paths for development and differentiation. Proc. Natl. Acad. Sci. USA. 108: 8257-8262.

[23] Zhang F, Xu L, Zhang K, Wang E, Wang J (2012) The potential and flux landscape theory of evolution. J. Chem. Phys. 137(6):065102.

[24] Qian H (2001) Mesoscopic nonequilibrium thermodynamics of single macromolecules and dynamic entropy-energy compensation. Phys Rev E 65(1):016102.

[25] Yoda M. Ushikubo T, amd Inoue W and Sasai M(2007)Roles of Roles of noise in single and coupled multiple genetic oscillators. J. Chem. Phys.126:115101,1-11.

[26] Mas-Colell A.(1986) The Price Equilibrium Existence Problem in Topological Vector Lattices. Econometrica 54(5):1039-1053. 
[27] Mankiw NG.(2011) Principles of Economics, 5th edition. South-western Cengage Learning.

[28] Weitzman ML (1974) Prices vs. Quantities. Reviews of Economic Studies 41:477-491.

[29] Woodcock A.E.R, Davis M (1978) Catastrophe Theory. New York:E.P.Dutton. 